

\title{
THREE-DIMENSIONAL BEM ANALYSIS TO ASSESS DELAMINATION CRACKS BETWEEN TWO TRANSVERSELY ISOTROPIC MATERIALS
}

\author{
Nicolás O. LARrosa, JHONny E. Ortiz And AdRián P. Cisilino
}

\begin{abstract}
Beyond the inherent attribute of reducing the dimensionality of the problem, the attraction of the boundary element method (BEM) for dealing with fracture mechanic problems is its accuracy in solving strong geometrical discontinuities. Within this context, a three-dimensional implementation of the energy domain integral (EDI) for the analysis of interface cracks in transversely isotropic bimaterials is presented in this paper. The EDI allows extending the two-dimensional $J$-integral to three dimensions by means of a domain representation naturally compatible with the BEM, in which the required stresses, strains, and derivatives of displacements are evaluated using their appropriate boundary integral equations. To this end, the BEM implementation uses a set of recently introduced fundamental solutions for transversely isotropic materials. Several examples are solved in order to demonstrate the efficiency and accuracy of the implementation for solving straight and curved crack-front problems.
\end{abstract}

\section{Introduction}

High-performance composite materials possess excellent mechanical properties such as strength, toughness, and fatigue resistance. Composite materials are ideal for components which require high strength per weight and stiffness per weight ratios. By choosing an appropriate combination of reinforcement and matrix material, manufacturers can produce materials with mechanical properties that fit the requirements for a particular purpose. Commonly, high strength and stiffness are required in various directions within a plane. A solution is to stack and weld together a number of plies, each having with the fibers oriented in a different direction. Such a stack is termed a laminate. The individual plies present a macroscopic transversely isotropic behavior with the symmetry axis in the direction of the fibers [Gibson 2007].

However, the application of composite materials in critical components has lagged due to the lack of sufficient knowledge about composite damage tolerance properties. Delamination, for example, is one of the areas that still demand a lot of work. Delamination consists in the nucleation of interface cracks between the plies of the laminate as a consequence of thermomechanical fatigue, impact, or material degradation [Gibson 2007]. Progress in the mechanics of interface fracture has been generally focused on the two-dimensional idealization of an interface crack, and not until recently has major effort been conducted on the three-dimensional aspect of interface fracture. That is in part due to the complexity of such problems and the very large computational effort required for their numerical analysis. However,

This work has been partially supported by the Agencia Nacional de Promoción Científica y Tecnológica (ANPCyT) of Argentina through grant PICT 2007 No. 1154. J. E. Ortiz has been supported by the Programa Ramón y Cajal of the Spanish Ministry of Science and Innovation.

Keywords: three-dimensional interface cracks, transversely isotropic bimaterials, energy domain integral, boundary element method. 
given the material mismatch at the interface boundary, it is expected that the three-dimensional effects play a more significant role in laminate structures than in homogeneous structures.

The numerical analysis of interface cracks in transversally isotropic materials has been traditionally addressed using finite element analysis; see, for example, [Boniface and Banks-Sills 2002; Freed and Banks-Sills 2005]. There is also the alternative of using the boundary element method (BEM). The attraction of the BEM can be largely attributed to the reduction in the dimensionality of the problem; this means that, compared to finite-element domain-type analysis, BEM analysis results in a substantial reduction in data preparation. At the same time, due to the inherent characteristics of its formulation, BEM provides very accurate results for problems containing strong geometrical discontinuities. Fracture mechanical analysis of three-dimensional transversely isotropic materials using BEM has been reported in [Sáez et al. 1997; Ariza and Dominguez 2004a; 2004b], which modeled static and dynamic crack problems, [Zhao et al. 2007], which derived the displacement discontinuity boundary integral equation, and more recently in [Chen et al. 2009], which studied the stress intensity factors of a central square crack in a transversely isotropic cuboid with arbitrary material orientations. To our knowledge, there is no published material about three-dimensional BEM modeling of interface cracks in dissimilar transversely isotropic bimaterials.

A number of techniques have been proposed for the evaluation of fracture parameters of interface cracks using FEM and BEM. They are, among others, the virtual crack extension approach [So et al. 2004], contour and domain path-independent integrals [Chow and Atluri 1998; Freed and Banks-Sills 2005; Ortiz and Cisilino 2005; Shah et al. 2006], displacement extrapolation techniques [Tan and Gao 1990; Mao and Sun 1995; Freed and Banks-Sills 2005], and special crack-tip elements [He et al. 1994]. In particular, path-independent integral techniques are derived from the $J$-integral proposed in [Rice 1968]. Being an energy approach, path-independent integrals eliminate the need to solve local crack tip fields accurately. If the integration domain is defined over a relatively large portion of the mesh, accurate modeling of the crack tip is unnecessary because the crack tip field contribution to the overall energy is not significant. At the same time, the $J$-integral approach developed in [Rice 1968] characterizes the crack driving force for two-dimensional problems. Therefore, for general three-dimensional cases involving cracks of arbitrary shape an alternative form for the $J$-integral is needed.

Three basic schemes have evolved for the numerical computation of the $J$-integral in three dimensions: virtual crack extension methods, generalization of Rice's contour integral, and domain integral methods [Anderson 2005]. Domain integrals are equivalent to the virtual crack extension technique and are better suited for numerical analysis than contour integral methods. Among the available domain integral methods (see for example, [Nikishkov and Atluri 1987; Feijoó et al. 2000]), the energy domain integral (EDI) of [Moran and Shih 1987] was chosen for this work.

The EDI can be formulated by applying the divergence theorem to Rice's $J$-integral. It produces a domain-independent integral defined over finite volumes enclosing some portion of the crack front [Moran and Shih 1987]. Previous works by the authors of this paper have demonstrated the versatility and efficiency of the BEM implementation of the EDI for assessing three-dimensional cracks in elastic [Cisilino et al. 1998], elastoplastic [Cisilino and Aliabadi 1999], and thermoelastic bodies [Balderrama et al. 2006; 2008] and for interface cracks in dissimilar isotropic bimaterials [Ortiz and Cisilino 2005].

This work introduces the BEM implementation of the EDI for the computation of the $J$-integral in three-dimensional interface cracks in transversely isotropic bimaterials. The BEM implementation uses 
the fundamental solutions recently introduced in [Távara et al. 2008]. The BEM solution strategy for the fracture problem and the EDI implementation is an extension of that proposed in [Ortiz and Cisilino 2005] for interface cracks in dissimilar isotropic bimaterials. Several examples are solved and the results compared to those available in the literature.

\section{Transversely isotropic materials}

The basic constitutive expressions governing the elastic behavior of transversely isotropic materials are reviewed, following [Ting 1996]. The general constitutive law of the anisotropic material is

$$
\sigma_{i j}(x)=C_{i j k l}(x) \varepsilon_{k l}(x)=C_{i j k l}(x) u_{k, l}(x),
$$

where, relative to a fixed rectangular Cartesian coordinate system, $\sigma_{i j}(x)$ are the components of the stress tensor, $\varepsilon_{i j}(x)$ are the components of the infinitesimal strain tensor, and $u_{k}(x)$ are the components of the displacement vector. Partial derivatives are indicated using comma notation. The $C_{i j k l}(x)$ are the components of the fourth-order constitutive tensor $C$, defined in terms of 21 independent elasticity constants.

Transversely isotropic materials are those with an axis of symmetry such that all directions perpendicular to that axis are on a plane of isotropy. In such a case the constitutive tensor can be defined in terms of only 5 independent elasticity constants. Using the Voigt reduced notation, the fourth-order constitutive tensor $C_{i j}(i, j=1, \ldots, 6)$ for a transversely isotropic material with the axis of symmetry coincident with the Cartesian axis $x_{3}$ can be expressed in terms of the following five elastic constants:

$$
C_{1111}=C_{11}, \quad C_{3333}=C_{33}, \quad C_{1122}=C_{12}, \quad C_{1133}=C_{13}, \quad C_{2323}=C_{44} .
$$

Due to symmetry with respect to $x_{3}, C_{66}=\left(C_{11}-C_{12}\right) / 2$.

The coefficients of the constitutive tensor $C_{i j}$ can be written in terms of the elastic engineering constants as follows:

$$
C_{11}=\frac{E\left(n-v^{\prime 2}\right)}{\lambda(1+v)}, \quad C_{12}=\frac{E\left(n+v^{\prime 2}\right)}{\lambda(1+v)}, \quad C_{13}=\frac{E v^{\prime}}{\lambda}, \quad C_{33}=\frac{E(1+v)}{\lambda}, \quad C_{44}=\mu^{\prime},
$$

where

$$
\lambda=n(1-v)-2 v^{\prime 2}, \quad n=E / E^{\prime},
$$

and

- $E$ and $E^{\prime}$ are the Young's moduli in the plane of isotropy and in the direction normal to it, respectively,

- $v$ is the Poisson's ratio that represents the strain response in the plane of isotropy due to an action parallel to it and $v^{\prime}$ is the lateral strain response for the planes normal to the plane of isotropy, and

- $\mu^{\prime}$ is the shear modulus for the planes normal to the planes of transverse isotropy.

\section{The energy domain integral}

Consider a three-dimensional crack front with a continuously turning tangent as depicted in Figure 1a with a local coordinate system $x^{*}$ at position $\eta$, given by $x_{1}^{*}$ normal to the crack front, $x_{2}^{*}$ normal to 


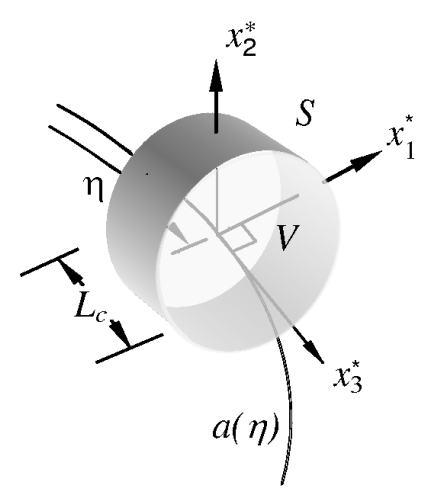

(a)

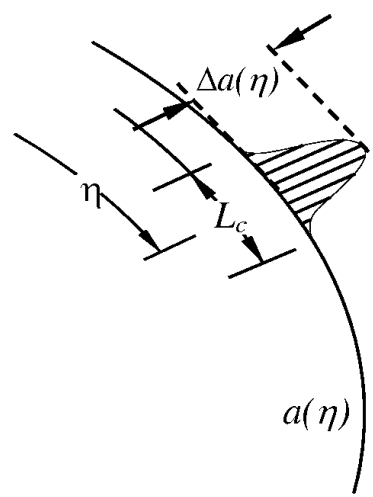

(b)

Figure 1. (a) Definition of the local orthogonal Cartesian coordinates at point $\eta$ on the crack front and integration volume, and (b) virtual crack front advance.

the crack plane, and $x_{3}^{*}$ tangent to the crack front. In addition, a tubular domain $V$ surrounds the crack segment $L_{c}$ which contains the position $\eta$.

Following [Nahta and Moran 1993], the $J$-integral at the position $\eta$ can be computed using the EDI:

$$
J(\eta)=\frac{\bar{G}(\eta)}{\int_{L_{c}} \Delta a(\eta) d l},
$$

where $\bar{G}(\eta)$ gives the total energy released when the finite segment $L_{c}$ undergoes the virtual displacement, $\Delta a(\eta)$, in the plane of the crack (see Figure 1b).

The expression of the energy release rate is

$$
\bar{G}(\eta)=\int_{V}\left(\sigma_{i j}^{*} u_{j, k}^{*}-w \cdot \delta_{k i}\right) q_{k, i} d V
$$

where $w$ is the strain energy density, $\sigma_{i j}^{*}$ and $u_{j, k}^{*}$ are Cartesian components of stress and displacement derivatives expressed in the system $x^{*}$, the integration domain $V$ is the volume of the tubular domain that surrounds the crack segment $L_{c}$, and $q$ is an auxiliary vector function used to represent the virtual crack advance as follows (see Figure 1b):

$$
q_{k}= \begin{cases}\Delta a(\eta) \cdot \xi_{k}(\eta) & \text { on } L_{c} \\ 0 & \text { on } S\end{cases}
$$

The function $q$ must be smooth in $V$, possess a maximum at the position $\eta$, and vanishes on the surfaces of $V$. The symbol $\xi_{k}(\eta)$ in (7) stands for the $k$-th component of the unit outward normal to the crack front in the crack plane $x_{1}^{*}-x_{3}^{*}$.

Analogously to the path-independence of its classical two-dimensional counterpart, the EDI formulation of the $J$-integral is independent of the integration volume $V$ [Nahta and Moran 1993]. 


\section{Boundary element formulation and implementation}

In order to account for the nonhomogeneous material properties, a multidomain BEM formulation is used for the problem solution. The modeling strategy is illustrated in the schematic representation in Figure 2 for a model consisting of two subdomains, $\Omega_{\mathrm{I}}(x)$ and $\Omega_{\mathrm{II}}(x)$, with external boundaries $\Gamma_{\mathrm{I}}(x)$ and $\Gamma_{\mathrm{II}}(x)$, respectively. Both subdomains share a common interface $\Gamma_{\mathrm{I}-\mathrm{II}}(x)$, a portion of which is debonded, and thus an interface crack is introduced. The subdomains possess linear transversely isotropic material behaviors as described in Section 2. The orientation of the material is specified using a local Cartesian system $\left(x_{1}^{0}, x_{2}^{0}, x_{3}^{0}\right)$ for each subdomain. In every case the direction of the symmetry axis of the material is chosen coincident with the direction $x_{3}^{0}$ (see Figure 2). In this way, it is possible to model interface cracks lying between laminates with arbitrary relative orientations.

The standard BEM uses the displacement boundary integral equation to relate the displacement and traction fields, $u(x)$ and $t(x)$, over the model boundary in the global coordinate system (see [Aliabadi 2002]):

$$
c_{i k}\left(x^{\prime}\right) u_{i}\left(x^{\prime}\right)+\int_{\Gamma} T_{i k}\left(x, x^{\prime}\right) u_{i}(x) d \Gamma(x)=\int_{\Gamma} U_{i k}\left(x, x^{\prime}\right) t_{i}(x) d \Gamma(x),
$$

where $U_{i k}\left(x, x^{\prime}\right)$ and $T_{i k}\left(x, x^{\prime}\right)$ are the displacement and traction fundamental solutions for transversely isotropic materials, respectively. The fundamental solutions account for the solution of the $i$-th component of the displacement and traction fields, $u_{i}(x)$ and $t_{i}(x)$, at the field point, $x$, due to the action of a unit load acting in the direction $j$ at the source point, $x^{\prime}$ (see the next section for the details about the fundamental solutions used in this work). The symbol $c_{i k}$ is the so-called jump term which depends on the local geometry at the source point, $x^{\prime}$, only.

BEM models are discretized using 9-node quadrilateral elements. Continuous elements are used everywhere in the model, except at the intersections of the interface and the crack faces with the model

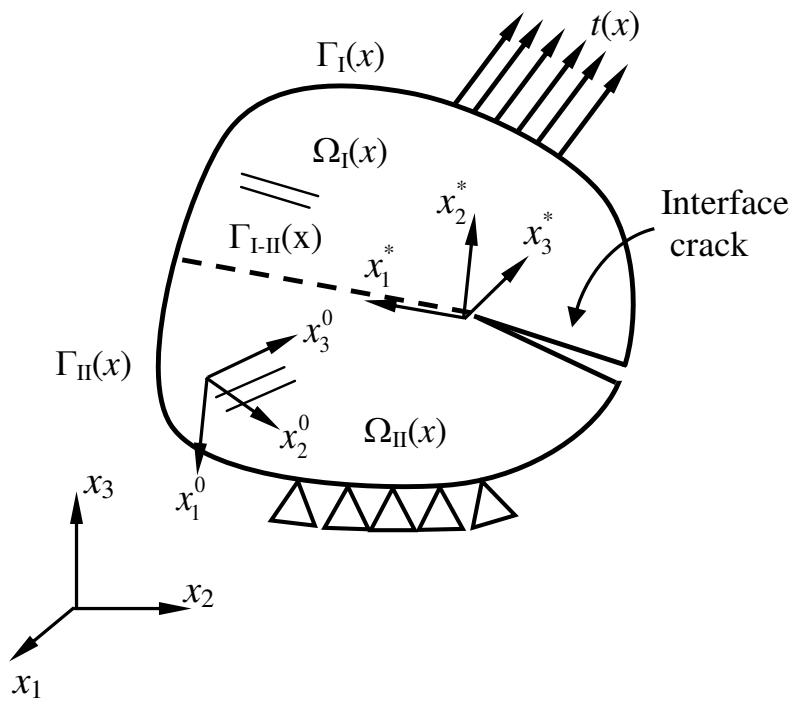

Figure 2. Schematic two-dimensional representation of the multidomain BEM model with an interface crack. 


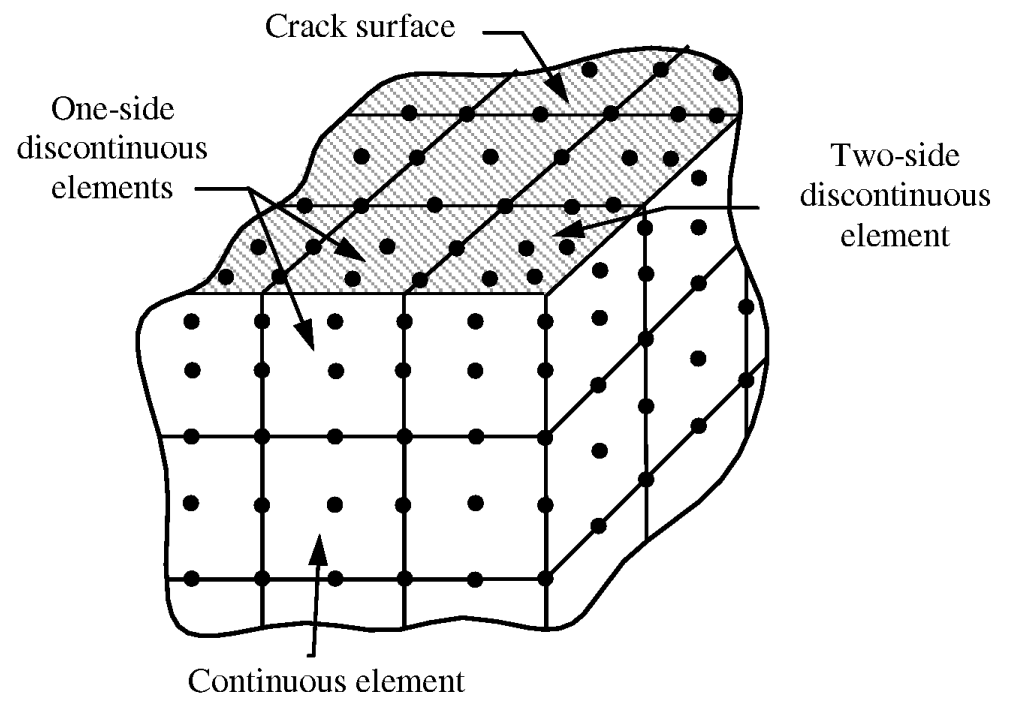

Figure 3. Model discretization strategy using continuous and one and two-side discontinuous elements.

outer surface. In such cases one and two-side discontinuous elements are used in order to avoid common nodes at the intersections (see Figure 3). It is worth noting that, although discontinuous elements are not strictly necessary to solve most of the practical bimaterial crack problems, they have been implemented in this work in order to develop a versatile and robust discretization strategy capable of dealing with general multiple subdomain problems (including the case of more than two subdomains sharing a single edge). At the same time, the implementation remains open to introduce further extensions to account for crack propagation which could require automatic model remeshing.

The regular BEM integrals over continuous and discontinuous elements are evaluated using standard Gaussian quadrature. In the case of nearly singular integrals an adaptive element subdivision technique is also employed. On the other hand, the Cauchy principal-value integrals and the free terms are evaluated using the rigid-body motion approach (see [Aliabadi 2002]). Singular integrals are computed using the variable transformation technique of [Lachat and Watson 1976].

The equilibrium and continuity conditions are enforced at the nodes lying on the interface $\Gamma_{\text {I-II }}$ shared by the two regions. In the case that no external forces are applied on the interface, the equilibrium condition is $t_{\mathrm{I}}=-t_{\mathrm{II}}$. The continuity condition is $u_{\mathrm{I}}=u_{\mathrm{II}}$. For further details on the multidomain BEM formulation and implementation the reader is referred to [Aliabadi 2002].

Comninou [1977] showed that the solution of the stress fields for a crack between dissimilar materials always predicts a contact zone between the crack surfaces at the crack tip. However, according to [Rice 1988] elastic fracture mechanics procedures are still valid when the inevitable nonlinear contact-zone size is small compared with the crack size. It is assumed in this work that this condition is always satisfied, and so the BEM implementation does not account for contact between the crack surfaces.

The computation of the $J$-integral is included in the BEM code as a postprocessing procedure, and so it could be applied to the results from a particular model at a later stage. The required stresses, strains, and derivatives of displacements at internal points are directly obtained from their boundary integral 
representations [Aliabadi 2002]:

$$
u_{i, m}\left(X^{\prime}\right)=\int_{\Gamma} U_{i j, m}\left(x, X^{\prime}\right) t_{j}(x) d \Gamma(x)-\int_{\Gamma} T_{i j, m}\left(x, X^{\prime}\right) u_{j}(x) d \Gamma(x),
$$

where $X^{\prime}$ is the coordinate of the internal point, and $U_{i j, m}\left(x, X^{\prime}\right)$ and $T_{i j, m}\left(x, X^{\prime}\right)$ are the derivatives of the fundamental displacement and traction fundamental solutions. The boundary $\Gamma$ corresponds to the boundary of the subdomain which the internal point $X^{\prime}$ lies on. Strains and stresses at internal points can then be easily computed using the definition of the infinitesimal strain tensor $\varepsilon_{i j}=\left(u_{i, j}+u_{j, i}\right) / 2$ and the constitutive relations in (1).

On the other hand, the derivatives of the displacements, strains, and displacements for boundary points are evaluated from the boundary displacements and tractions by means of a procedure similar to that usually used for finite elements. For further details the reader is referred to [Ortiz and Cisilino 2005].

Finally, in order to proceed with the $J$-integral computation, the resultant displacement derivatives, strains, and stresses for both internal and boundary points are transformed to the local crack-front coordinate system $\left(x_{1}^{*}, x_{2}^{*}, x_{3}^{*}\right)$ introduced in Section 3 using the standard transformation rule for second-order tensors; see [Ting 1996].

\section{The fundamental solutions for transversely isotropic materials}

There are several expressions for the fundamental solutions for transversely isotropic materials; see, for example, [Pan and Chou 1976; Loloi 2000]. However, these solutions could be cumbersome to implement in a BEM code because of the multiple cases to consider due to all the possible material orientations and the relative positions of the source and field points.

Távara et al. [2008] have recently derived completely general and unique expressions valid for all possible configurations given in terms of real functions only. These fundamental solutions are presented in what follows.

The Green's function for a linearly elastic anisotropic medium using the Barnett-Lothe tensor is [Lifshitz and Rozentsveig 1947]

$$
U^{0}(x)=\frac{1}{4 \pi r} H(x)
$$

where $x$ is the position vector and the matrix $H(x)$ defined by

$$
H(x)=\frac{1}{\pi} \int_{-\infty}^{+\infty} \Gamma^{-1}(p) d p .
$$

The integrand $\Gamma(p)$ is the $3 \times 3$ matrix

$$
\Gamma(p)=Q+p\left(R+R^{T}\right)+p^{2} T,
$$

where superscript $T$ means transpose, and the matrices $Q, R$, and $T$ are defined by

$$
Q_{i j}=C_{i j k s} n_{j} n_{s}, \quad R_{i k}=C_{i j k s} n_{j} m_{s}, \quad T_{i k}=C_{i j k s} m_{j} m_{s},
$$

for $n$ and $m$ orthogonal unit vectors in the plane normal to the position vector $x$. The matrices $Q$ and $T$ are symmetric and positive definite if the deformation energy of the material is positive. Considering (12) and (13), the matrix $H(x)$ is also symmetric and depends on the direction of the position vector $x$, 
but not on its magnitude. Using residues theory, $H(x)$ in (11) can be expressed in the same form as (10) and (12):

$$
H(x)=2 i \sum_{v=1}^{3} \frac{\hat{\Gamma}\left(p_{v}\right)}{|\Gamma(p)|^{\prime}},
$$

where $\hat{\Gamma}\left(p_{v}\right)$ is the adjunct of the matrix of $\Gamma(p)$ defined in (12) and $|\Gamma(p)|$ is its determinant. The values $p_{v}$ are the so-called Stroh eigenvalues of the sextic equation:

$$
|\Gamma(p)|=0
$$

The eigenvalues of (15) can be represented as $p_{v}=\alpha_{v}+i \beta_{v}$ where both $\alpha_{v}$ and $\beta_{v}$ are real with $\beta_{v}>0$ $(v=1,2,3)$. Although there are explicit solutions for (14) in terms of the eigenvalues, $p_{v}$, and for the Green's function in (10), they are not of practical use here because they are not general and they do not hold for the degenerate cases $p_{1}=p_{2}$ and $p_{1}=p_{2}=p_{3}$. Alternatively, a simplified solution for (14) can be obtained when (15) is a cubic equation in $p^{2}$ of the form

$$
|\Gamma(p)|=|T|\left(p^{2}-p_{1}^{2}\right)\left(p^{2}-p_{2}^{2}\right)\left(p^{2}-p_{3}^{2}\right) .
$$

In this case, (15) can be expressed as

$$
\left.\left[p^{4}+\left(g^{2}-2 h\right) p^{2}+h^{2}\right)\right]\left[p^{2}+\beta_{3}^{2}\right]=0,
$$

where $g, h$, and $\beta_{3}$ are defined in the Appendix.

A new expression for $H(x)$ is obtained for any anisotropic linear elastic material

$$
H(x)=\frac{1}{|T|} \sum_{n=0}^{4} p^{n} \hat{\Gamma}^{(n)} .
$$

Using (16) and (18) with (14), the following expression results:

$$
H(x)=\frac{1}{|T| \xi}\left\{\frac{\zeta}{h \beta_{3}} \hat{\Gamma}^{(0)}+\hat{\Gamma}^{(2)}+\delta \hat{\Gamma}^{(4)}\right\}
$$

where

$$
\begin{aligned}
& \zeta=-i\left(p_{1}+p_{2}+p_{3}\right)=g+\beta_{3}, \\
& \delta=-\left(p_{1} p_{2}+p_{2} p_{3}+p_{3} p_{1}\right)=h+g \beta_{3}, \\
& \xi=i\left(p_{1}+p_{2}\right)\left(p_{2}+p_{3}\right)\left(p_{1}+p_{3}\right)=g\left(h+g \beta_{3}+\beta_{3}^{2}\right) .
\end{aligned}
$$

The terms $\zeta, \delta$, and $\xi$ depend only on $p_{1}+p_{2}, p_{1} p_{2}$, and $p_{3}$; therefore, it is not necessary to calculate all the eigenvalues. The solution to (19) is valid for degenerate and nondegenerate cases. The terms $\beta_{3}$, $h$, and $g$ can be computed using (17).

A relatively simple and general expression for $H(x)$ for transversely isotropic materials can be obtained using the auxiliary vector $\hat{x}=\left(r_{12}, 0, x_{3}^{2}\right)$, where $r_{12}=\left[\left(x_{1}^{0}\right)^{2}+\left(x_{2}^{0}\right)^{2}\right]^{1 / 2},\left(x_{1}^{0}, x_{2}^{0}, x_{3}^{0}\right)$ is the local coordinate system, and the triad $[n, m, \hat{x} / r]$, with $n=(c, 0,-s)$ and $m=(0,1,0)$, where $c=\cos \phi=x_{3}^{0} / r$, 


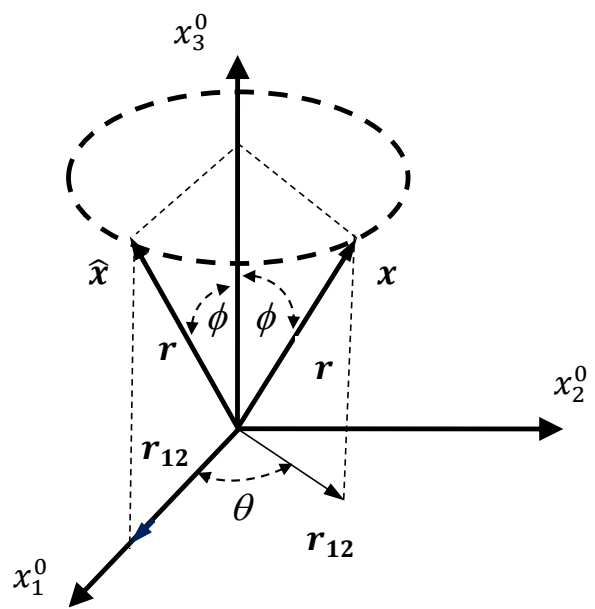

Figure 4. Points $\boldsymbol{x}$ and $\hat{\boldsymbol{x}}$ associated with a transversely isotropic material.

$s=\sin \phi=r_{12} / r$, and $0 \leq \phi \leq \pi$, is illustrated in Figure 4. For such a coordinate system, the only nonzero coefficients are given by

$$
\begin{array}{lll}
H_{11}=\frac{1}{C_{66} \beta_{3}}+\frac{C_{44} c^{2}+C_{33} s^{2}}{C_{11} C_{44} g h}-\frac{f}{\xi}, & H_{22}=\frac{1}{C_{11} g}+\frac{f}{\xi}, \\
H_{33}=\frac{1}{g h}\left\{\frac{h+c^{2}}{C_{44}}+\frac{s^{2}}{C_{11}}\right\}, & H_{13}=\frac{\left(C_{13}+C_{44}\right) s c}{C_{11} C_{44} g h} .
\end{array}
$$

The additional terms in (23) are given in the Appendix. The general expression of the tensor $H(x)$ for any $x$ can be obtained by transformation of the components:

$$
H_{i j}(x)=\Omega_{i k} \Omega_{j s} H_{k s}(\hat{x}),
$$

where the rotation matrix $\Omega_{i j}$ is

$$
\Omega_{i j}=\left(\begin{array}{ccc}
\cos \theta & -\sin \theta & 0 \\
\sin \theta & \cos \theta & 0 \\
0 & 0 & 1
\end{array}\right)
$$

The derivatives of the fundamental solution for the displacement can be expressed using the modulation function $\hat{U}_{i j, k}(x)$ :

$$
U_{i j, k}^{0}(x)=\frac{\hat{U}_{i j, k}(x)}{4 \pi r^{2}} .
$$

$\hat{U}_{i j, k}(x)$ is an odd function, which depends on the direction of $x$ but not on its magnitude, that is, $\hat{U}_{i j, k}(x)=-\hat{U}_{i j, k}(-x / r)$. Using the transformation in (24), the derivatives of the displacement fundamental solution are:

$$
\hat{U}_{i j, k}(x)=\Omega_{i a} \Omega_{j b} \Omega_{k c} \hat{U}_{a b, c}(\hat{x}) .
$$

The closed-form expressions of $\hat{U}_{i j, k}(\hat{x})$ can be found in [Távara et al. 2008]. 
The fundamental solution for the stresses, $\Sigma_{i j, k}(x)$, can be obtained by applying Hooke's law for transversely isotropic material, yielding

$$
\Sigma_{i j k}^{0}(x)=\frac{\hat{\Sigma}_{i j k}(x)}{4 \pi r^{2}},
$$

where $\hat{\Sigma}_{i j, k}(x)$ is an odd symmetric function. So, the stress fundamental solution can be expressed in a similar form to (27):

$$
\hat{\Sigma}_{i j k}(x)=\Omega_{i a} \Omega_{j b} \Omega_{k c} \hat{\Sigma}_{a b c}(\hat{x}) .
$$

The closed-form expressions of $\hat{\Sigma}_{i j k}(\hat{x})$ can be found in [Távara et al. 2008]. The traction fundamental solution associated with the normal vector $n_{j}(x)$ can be obtained directly using

$$
T_{i k}^{o}(x)=\Sigma_{i j k}^{0}(x) n_{j}(x) .
$$

Finally, the fundamental solutions $U_{i k}(x)$ and $T_{i k}(x)$ have to be transformed from the local coordinate system, $\left(x_{1}^{0}, x_{2}^{0}, x_{3}^{0}\right)$, to the global in order to assemble the boundary integral (8). The fundamental solutions are transformed from the local coordinate system to the global via the standard transformations for second order tensors (see [Ting 1996]):

$$
U_{i j}(x)=a_{i k} a_{j l} U_{k l}^{o}(x), \quad T_{i j}(x)=a_{i k} a_{j l} T_{k l}^{o}(x),
$$

where $a_{i k}$ are the coefficients of the transformation matrix.

\section{6. $J$-integral computation}

The computation of the EDI was included in the BEM code as a postprocessing procedure, and so it could be applied to the results from a particular model at a later stage. As stated in Section 3, (5) allows the computation of the $J$-integral at any position $\boldsymbol{\eta}$ on the crack front. In each case, this requires the evaluation of a volume integral over a domain that encloses a segment of the crack front, $L_{c}$. A natural choice here is to make $\boldsymbol{\eta}$ coincident with the element nodes on the crack front, while $L_{c}$ is taken as the element or element sides where the point $\boldsymbol{\eta}$ lies. As depicted in Figure 5, three different cases are considered depending on whether the node $M$ at the location of the crack front position $\eta$ is a midside node, is shared by two elements, or is located coincident with the external surface (a surface node). If the

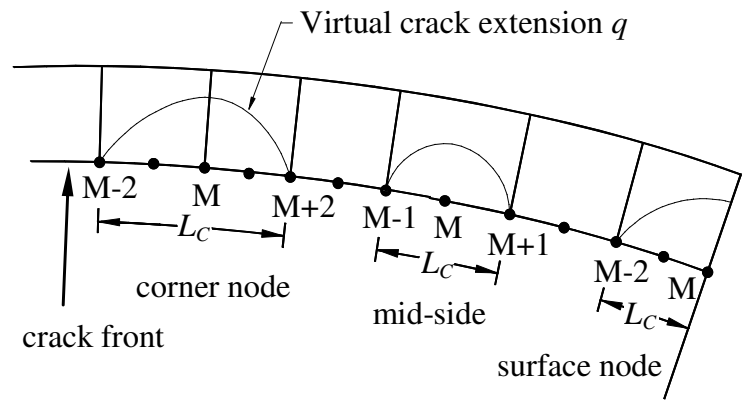

Figure 5. Schematic of the crack front region illustrating the $q$-function assimilated to the virtual crack extensions for a corner node, a midnode, and a surface node. 


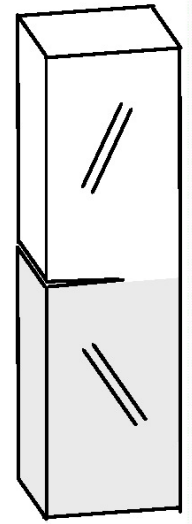

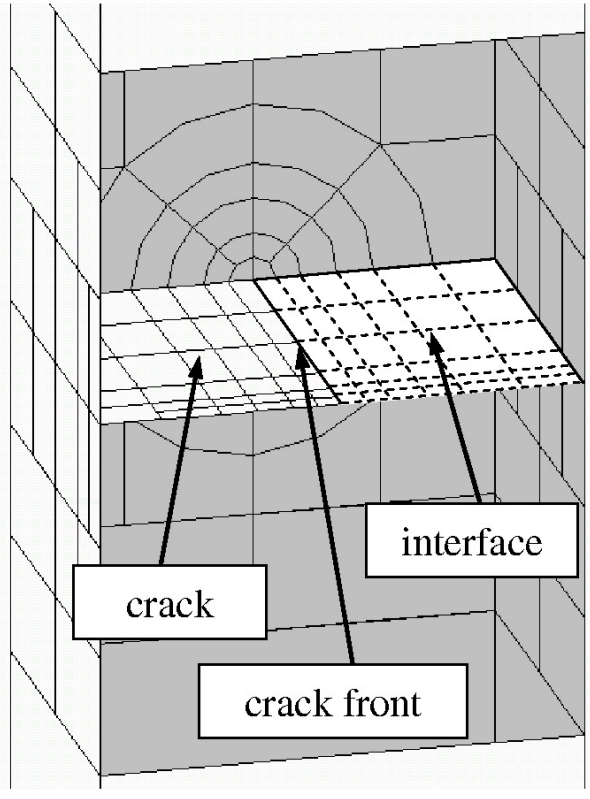

(b)

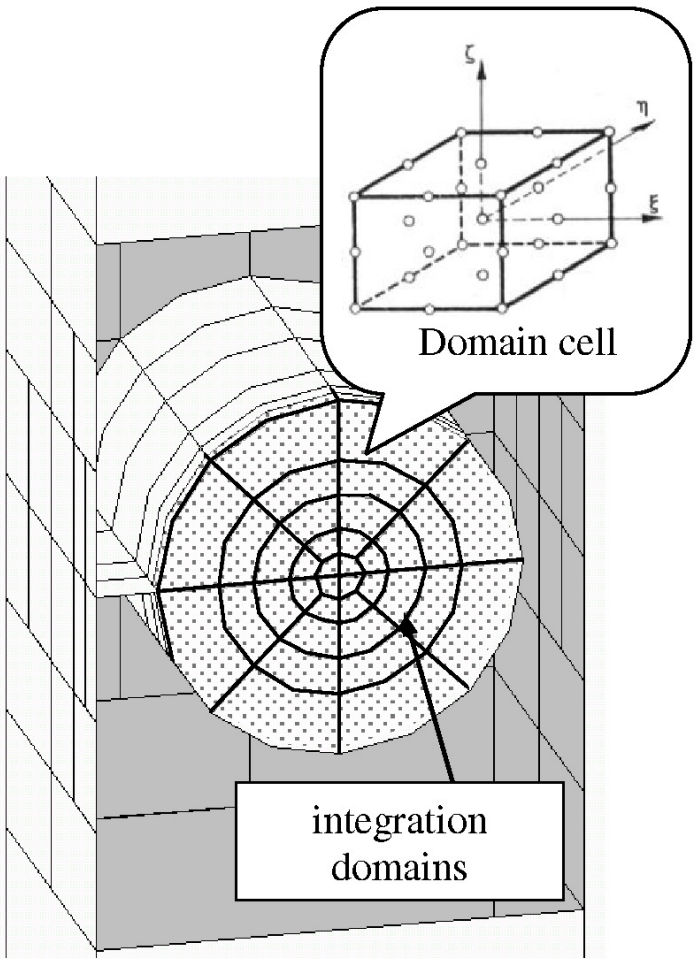

(c)

Figure 6. (a) Problem geometry, (b) boundary element discretization, and (c) integration domains. Note in (c) the detail illustrating the 27-node isoparametric (brick) cells. The dotted region indicates the volume cells defining the integration volumes used for the computation of the $J$-integral for the crack-front node on the specimen surface (see surface node in Figure 5).

node $M$ is a midside node or surface node, $L_{c}$ (the width of the integration domain) spans one element, connecting nodes $M-1, M$, and $M+1$ and nodes $M-2, M-1$, and $M$, respectively. On the other hand, if node $M$ is shared by two elements, $L_{c}$ spans both elements, connecting nodes from $M-2$ to $M+2$.

The boundary mesh is designed to have a web pattern around the crack front in order to build the integration domains for the evaluation of the EDI in the shape of cylinders. The integration volumes are discretized using 27-node isoparametric (brick) cells. This is illustrated in Figure 6, where a portion of the model surface has been removed to show the discretizations of the crack and of the integration domains.

Stresses, strains, and derivatives of displacements at cell nodes are computed using the procedures introduced in Section 4, and then transformed to the local crack-front coordinate system, $\left(x_{1}^{*}, x_{2}^{*}, x_{3}^{*}\right)$, using the standard transformation rule for second-order tensors. The stresses, strains, and displacements derivatives are approximated within cell domains by products of the cell interpolation functions, $\psi_{i}$, and the nodal values of $\sigma_{i j}^{*}, \varepsilon_{i j}^{*}$, and $u_{i, m}^{*}$. 
A central point in the implementation of the EDI is the specification of the values for the function $q$. Following the definition introduced in (7), the value of $q_{k}$ is specified equal to one for the cell node coincident with the position $\boldsymbol{\eta}$ on the crack front where the EDI is evaluated, and equal to zero for all the cell nodes located on the surface of the integration volume. For the implementation used in this work, the function $q$ is chosen to vary quadratically in the directions tangential and normal to the crack front: this is schematically illustrated in Figure 5 for the tangential direction on the $x_{1}^{*} x_{3}^{*}$-plane. This biquadratic definition of $q$ has been employed with excellent results in the computation of EDI for a variety of problems in previous works [Cisilino et al. 1998; Cisilino and Aliabadi 1999; Ortiz and Cisilino 2005; Balderrama et al. 2006; 2008]. Thus, considering that the evaluation point $\boldsymbol{\eta}$ is at the middle of the crack front segment, $L_{c}$, and that $r_{0}$ is the radius of the integration domain, the function $q$ is written as

$$
q\left(x^{*}\right)=\left|1-\left(\frac{x_{3}^{*}}{L_{c} / 2}\right)^{2}\right| \cdot\left[1-\left(\frac{r}{r_{0}}\right)^{2}\right],
$$

where $r$ is the distance from the crack front in the $x_{1}^{*} x_{3}^{*}$-plane (see Figure 1).

Equation (32) is used to specify the value of $q$ for all the cell nodes within the integration domain. Then, consistent with the isoparametric formulation, the $q$-values are interpolated within each volume cell using

$$
q=\sum_{i=1}^{27} \psi_{i} Q^{i}
$$

where the $\psi_{i}$ are the shape functions and $Q^{i}$ is the $q$-value for the $i$-th node. Following standard manipulations, the derivatives of $q$ are

$$
q_{k, j}=\sum_{i=1}^{27} \sum_{l=1}^{3} \frac{\partial \psi_{i}}{\partial \zeta_{l}} \frac{\partial \zeta_{l}}{\partial x_{j}^{*}} Q^{i}
$$

where $\zeta_{k}$ are the coordinates in the cell isoparametric space and $\partial \zeta_{k} / \partial x_{j}^{*}$ is the Jacobian matrix of the transformation.

Finally, if Gaussian integration is used, the discretized form of (6) is

$$
\bar{G}(\eta)=\sum_{\text {cells in } V} \sum_{p=1}^{m}\left\{\left(\sigma_{i j}^{*} u_{j, k}^{*}-\sigma_{i j}^{*} \varepsilon_{i j}^{*} \delta_{k i}\right) q_{k, j} \operatorname{det}\left(\frac{\partial x_{j}}{\partial \zeta_{k}}\right)\right\}_{p} w_{p},
$$

where $m$ is the number of Gaussian points per cell and the $w_{p}$ are their weighting factors.

\section{Application examples}

7.1. Thick bimaterial plate in tension with a center interface crack. A thick bimaterial plate containing a through crack on the interface is considered in the first example. A schematic representation of the problem geometry, dimensions, and boundary conditions is depicted in Figure 7. The model discretization is similar to that depicted in Figure 6. It consists of 658 elements and 2855 nodes. Eighteen elements are placed along the crack front and a total of 126 elements are used in the crack discretization. Five rings of cells with normalized radii $r / a=0.1,0.2,0.3,0.44$, and 0.64 are used around the crack front for $J$ computations. For this purpose 648 cells and 6438 nodes are employed. 


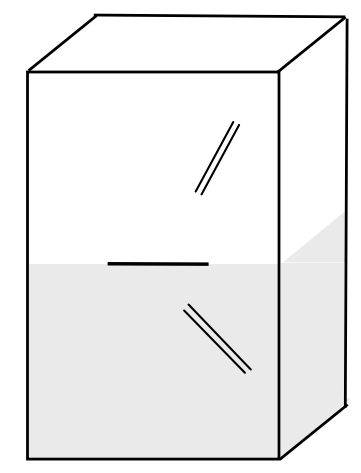

(a)

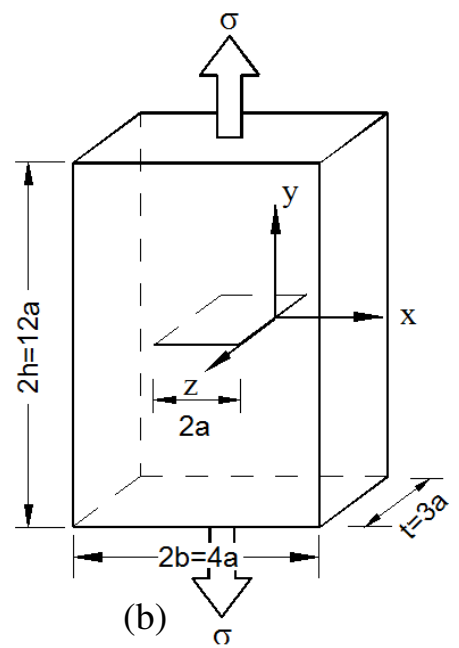

Figure 7. (a) Schematic representation of a thick tension plate with a center interface crack and (b) model dimensions.

In order to validate the code, the problem was solved first for homogeneous cases, that is, the material elastic constants and orientations were set the same for both subdomains. Thus, the direction of the axis of symmetry, $x_{3}^{0}$, was chosen to be parallel to the crack plane, that is, coincident with the global directions $x$ and $z$. The material elastic constants were chosen to be the same as those used for the laminated case in [Ariza and Dominguez 2004b]. The five independent values of the elastic constants $C_{i j}$ in (3) are

$$
C_{11}=5.37 \mathrm{GPa}, \quad C_{12}=1.34 \mathrm{GPa}, \quad C_{13}=3.35 \mathrm{GPa}, \quad C_{33}=251.168 \mathrm{GPa}, \quad C_{44}=5 \mathrm{GPa} .
$$

The associated elastic properties are: $E=5 \mathrm{GPa}, E^{\prime}=247.83 \mathrm{GPa}, v=0.245, v^{\prime}=0.01$, and $\mu^{\prime}=2.5$. The material orientation is specified for each subdomain by means of the angles which define the orientation of the material axis of symmetry, $x_{3}^{0}$, with respect to the global coordinate system $(x, y, z)$. In this way, for the material axis of symmetry oriented in the global direction $x$, the orientation angles are $0^{\circ} / 90^{\circ} / 90^{\circ}$, while for the material axis of symmetry oriented in the global direction $z$, the angles are $90^{\circ} / 90^{\circ} / 0^{\circ}$.

The computed results along the crack front are presented in Figure 8. In order to compare with other results, the data in Figure 8 is presented in terms of normalized stress intensity factors (SIF), $K_{I} / K_{0}$, where $K_{0}=\sigma \sqrt{\pi a}$. To compute the SIF from the $J$ results the expressions of [Chu and Hong 1990] are used:

$$
\begin{aligned}
& J_{1}=a_{11} K_{\mathrm{I}}^{2}+a_{12} K_{\mathrm{I}} K_{\mathrm{II}}+a_{22} K_{\mathrm{II}}^{2}, \\
& J_{2}=b_{11} K_{\mathrm{I}}^{2}+b_{12} K_{\mathrm{I}} K_{\mathrm{II}}+b_{22} K_{\mathrm{II}}^{2},
\end{aligned}
$$

where the coefficients $a_{i j}$ and $b_{i j}$ depend on the elastic material properties and the material orientation. The coefficients $a_{12}$ and $a_{22}$ and the three coefficients $b_{i j}$ are zero when one of the principal axes of the material is parallel to the crack plane. Thus, for the cases considered in this work,

$$
J_{1}=a_{11} K_{\mathrm{I}}^{2}
$$


These are the values of the coefficient $a_{11}$ and the ratio $E_{x} / E_{y}$ between Young's moduli:

$$
\begin{array}{lll}
\text { Material symmetry in } x \text {-direction: } & a_{11}=0.112 \times 10^{-9} & E_{x} / E_{y}=49.57 \\
\text { Material symmetry in z-direction: } & a_{11}=0.2 \times 10^{-9} & E_{x} / E_{y}=1
\end{array}
$$

It can be seen in Figure 8 that, excepting only the regions next to the lateral faces of the specimen (say, $|z / t|>0.45)$ where the boundary layer effect takes place, the SIF value is nearly constant along the crack front. Two sets of results obtained from a two-dimensional high-resolution finite element model are also shown in Figure 8. The finite element model was solved using ABAQUS [2009], and was discretized using a fine regular mesh consisting of 9,600 8-node biquadratic, plane stress elements (CPS8R). The SIF were computed using the ABAQUS built-in $J$-integral procedure. The resultant normalized SIF are $K_{I} / K_{0}=1.124$ and $K_{I} / K_{0}=1.184$ for the material axis of symmetry oriented in the global directions $x$ and $z$, respectively. The maximum difference between the BEM and FEM results along the crack front in the interior of the specimen (that is, excluding the regions next to the lateral faces) is less than $2 \%$.

The final case consists of a heterogeneous plate with the axis of symmetry of the material oriented in the global directions $z$ and $y$ for subdomains I and II, respectively; that is, $90^{\circ} / 90^{\circ} / 0^{\circ}$ for subdomain I and $90^{\circ} / 0^{\circ} / 90^{\circ}$ for subdomain II. The material elastic properties are the same as in the previous cases. Computed results are presented in Table 1. The results are normalized with respect to $J_{0}=\sigma^{2} \pi a / E^{\prime}$. It can be seen that the $J$ value is nearly constant along the complete crack front. Besides, the path independence is found to be excellent, with a standard deviation of around 5\% for the results computed using the domains with radii $r / a \geq 0.20$. The only exceptions are the positions next to the lateral face of

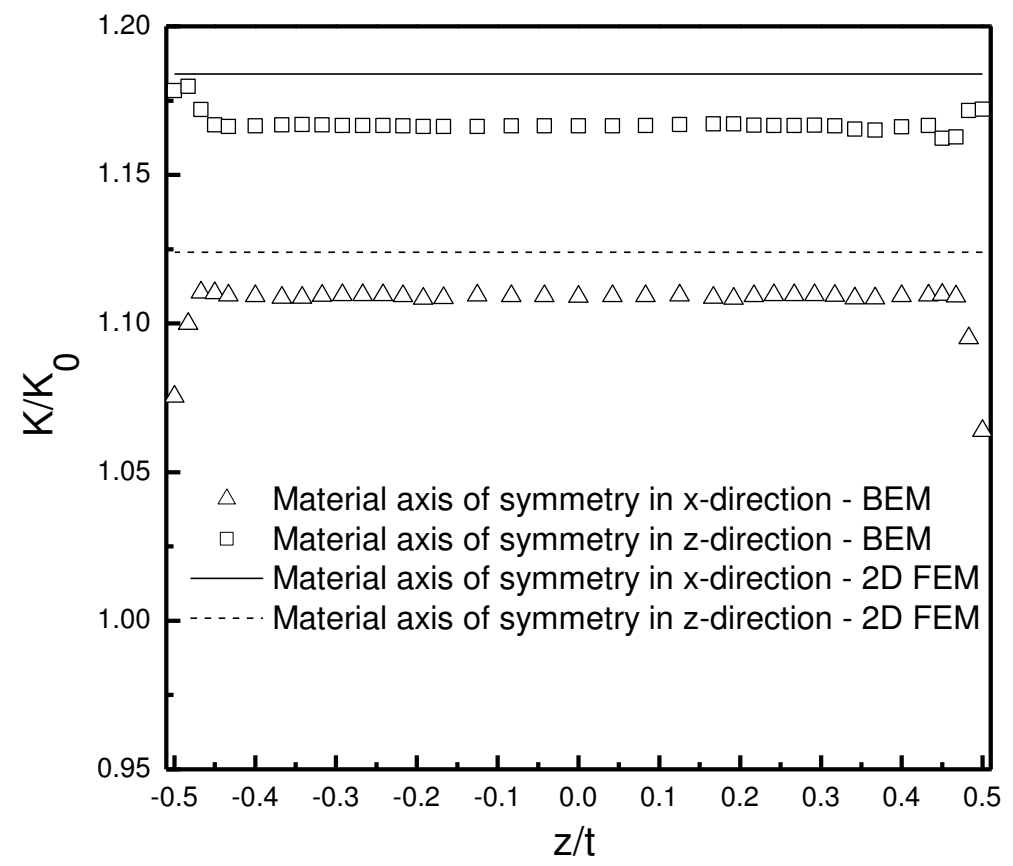

Figure 8. Normalized SIF results along the crack front for homogeneous transversely isotropic center crack specimen. 


\begin{tabular}{|cccccccc|}
\hline \multicolumn{7}{c|}{$r / a=$} \\
$z / t$ & 0.10 & 0.20 & 0.30 & 0.44 & 0.64 & Average & $\begin{array}{c}\text { Std. } \\
\text { dev. }\end{array}$ \\
0.000 & 25.6382 & 30.4503 & 30.7658 & 30.7658 & 30.6081 & 30.5613 & 0.49 \\
0.042 & 25.6382 & 30.4503 & 30.7658 & 30.7658 & 30.6081 & 30.5566 & 0.49 \\
0.083 & 25.6382 & 30.4503 & 30.7658 & 30.7658 & 30.6081 & 30.5512 & 0.49 \\
0.125 & 25.6382 & 30.4503 & 30.7658 & 30.7658 & 30.6081 & 30.5452 & 0.49 \\
0.167 & 25.6382 & 30.4503 & 30.7658 & 30.7658 & 30.6081 & 30.5384 & 0.49 \\
0.192 & 25.6382 & 30.4503 & 30.6869 & 30.7658 & 30.6081 & 30.5306 & 0.44 \\
0.217 & 25.6382 & 30.4503 & 30.6869 & 30.7658 & 30.6081 & 30.5231 & 0.44 \\
0.242 & 25.6382 & 30.4503 & 30.6869 & 30.7658 & 30.6081 & 30.5144 & 0.44 \\
0.267 & 25.6382 & 30.4503 & 30.6869 & 30.7658 & 30.6081 & 30.5041 & 0.44 \\
0.292 & 25.6382 & 30.4503 & 30.6869 & 30.7658 & 30.6081 & 30.4917 & 0.44 \\
0.317 & 25.6382 & 30.3714 & 30.6869 & 30.6869 & 30.5292 & 30.4766 & 0.50 \\
0.342 & 25.6382 & 30.3714 & 30.6081 & 30.6869 & 30.4503 & 30.4651 & 0.47 \\
0.367 & 25.6382 & 30.3714 & 30.6081 & 30.6081 & 30.4503 & 30.4559 & 0.39 \\
0.400 & 25.5593 & 30.3714 & 30.6081 & 30.6081 & 30.4503 & 30.4470 & 0.39 \\
0.433 & 25.5593 & 30.2925 & 30.6081 & 30.6081 & 30.4503 & 30.4345 & 0.50 \\
0.450 & 25.5593 & 30.2925 & 30.5292 & 30.6081 & 30.4503 & 30.4207 & 0.44 \\
0.467 & 25.4804 & 30.2925 & 30.6869 & 30.7658 & 30.6869 & 30.4043 & 0.70 \\
0.483 & 25.0071 & 29.9770 & 30.7658 & 31.1603 & 31.2391 & 30.3024 & 1.91 \\
0.500 & 23.5871 & 28.5570 & 29.7403 & 30.3714 & 30.6081 & 29.8192 & 3.08 \\
\hline
\end{tabular}

Table 1. Normalized $J$-integral results for the heterogeneous plate as a function of the integration domain size. The results for the smallest integration domains, $r / a=0.1$ (shaded column in the table) are excluded for the computation of the average value and the standard deviation.

the specimen, where the boundary layer effect takes place and the applicability of the $J$-integral is not strictly valid. The smallest integration domains with $r / a=0.10$ do not provide accurate results. This is attributed to the fact that these domains are discretized using a single cell in the radial direction. Similar behaviors were found by the authors previously [Cisilino et al. 1998; Ortiz and Cisilino 2005].

7.2. Bimaterial laminate with an edge interface crack. In this example the analysis of an edge crack in a bimaterial laminate is considered. The model geometry and discretization are shown in Figure 9. The model dimensions are: crack length $a=10 \mathrm{~mm}$, specimen width $b=4 a$, height $h=a$, and thickness $2 t=1.5 a$. The material properties are the same ones used in the previous example. The discretization consists of 596 elements. Five rings of cells with normalized radii $r / a=0.05,0.1,0.15,0.22$, and 0.32 are used around the crack front for the $J$ computations. 504 cells are used in the construction of the integration domains.

The model was solved for a number of relative orientations of the axis of symmetry of the material in both subdomains. The computed results are reported in Figure 10. The $J$-results in Figure 10 are normalized with respect to $J_{0}=\sigma^{2} \pi a / E^{\prime}$. It can be seen that when one of the principal axes of the material is specified perpendicular to the crack front direction for both subdomains, the $J$-integral results 


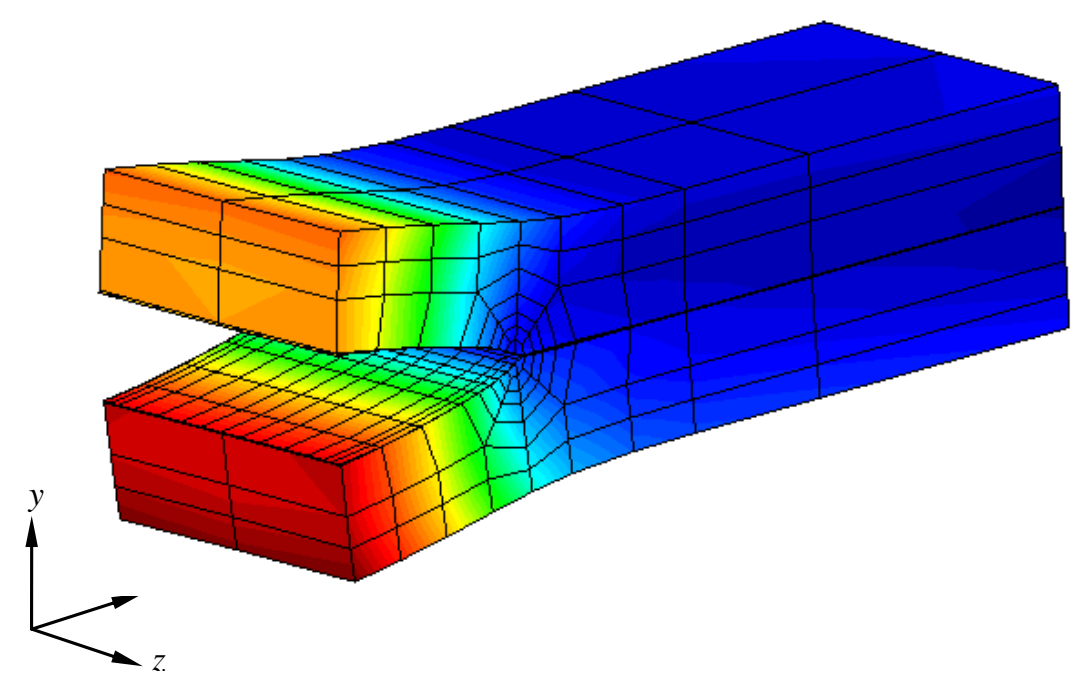

Figure 9. Bimaterial laminate with an edge crack (deformed geometry).

along the crack front are symmetric with respect to the specimen midplane $(z / t=0)$. This is the case for the results labeled $90^{\circ} / 90^{\circ} / 0^{\circ}-90^{\circ} / 90^{\circ} / 0^{\circ}$ and $0^{\circ} / 90^{\circ} / 90^{\circ}-90^{\circ} / 90^{\circ} / 0^{\circ}$ in the figure. On the other hand, when there is no principal axis of the material oriented perpendicular to the crack front in at least one of the subdomains, the $J$-integral results are not symmetric with respect to the specimen midplane. The extreme values for the $J$-integral are attained at the free surface.

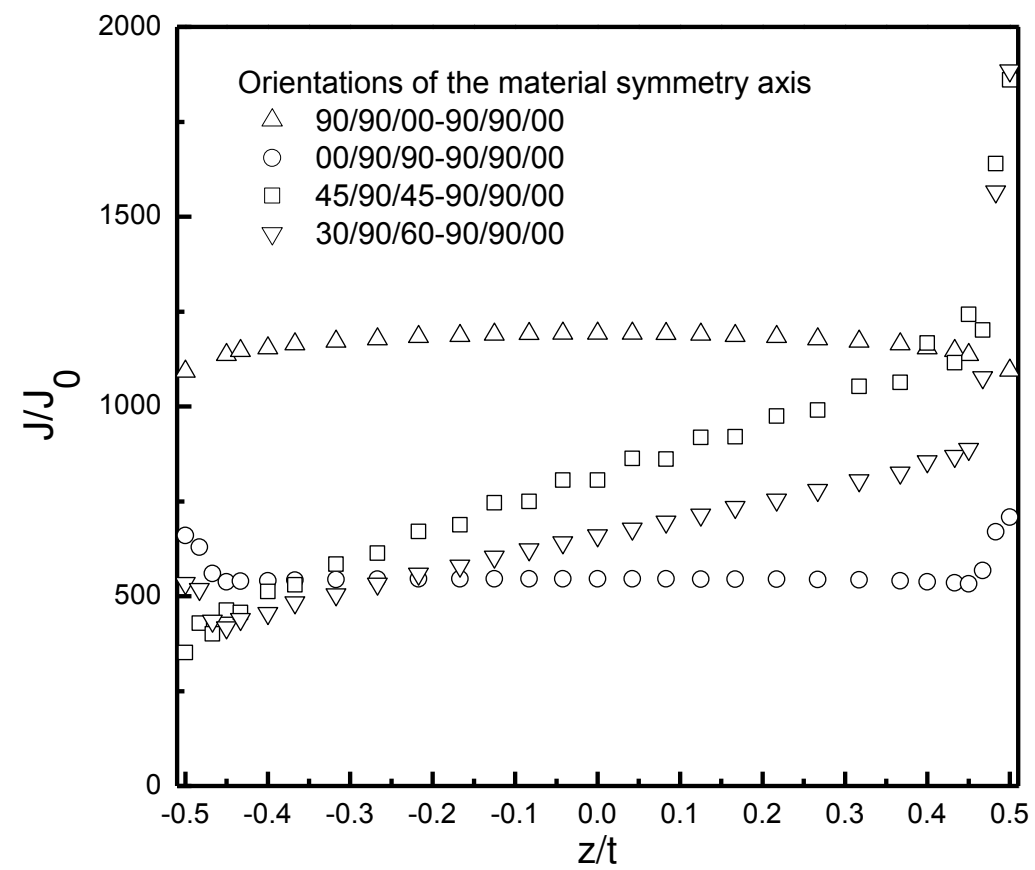

Figure 10. Normalized $J$-integral results along the crack front of the edge crack in the ply. 


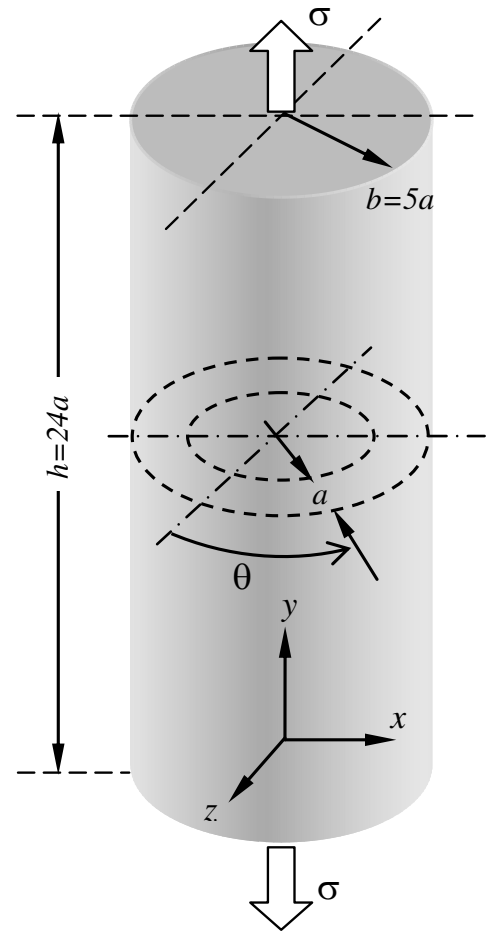

(a)

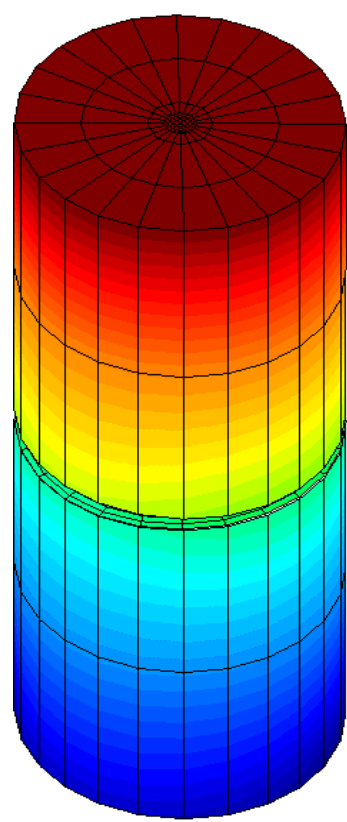

(b)

Figure 11. External circumferential interface crack in a cylindrical bimaterial bar. (a) Model geometry and dimensions and (b) model discretization (deformed mesh).

7.3. A circumferential interface crack in a cylindrical bimaterial bar. The last example consists of a cylindrical bimaterial bar containing a circumferential crack subjected to remote axial tension $\sigma$, as illustrated in Figure 11a. The radius of the bar is $b=5 a$ and its height is $h=24 a$, with $a$ being the crack depth. A total of 684 elements are employed in the model discretization. Four rings of cells with radii $r / a=0.25,0.5,0.75$, and 1 are used around the crack front for the $J$ computations. The integration domains are constructed using 672 cells. The model discretization is illustrated in Figure 11b. The material properties are the same reported for the previous examples.

The problem was solved considering different material orientations. The results are shown in Figure 12. In every case the results are normalized with respect to $J_{0}=\sigma^{2} \pi a / E^{\prime}$. The first solution is for an isotropic homogeneous material and was used for validation purposes. The $J$ result is constant along the complete crack front. Also plotted in Figure 12 is the result of [Murakami and Okazaki 1976], which possesses a reported accuracy of 3\%. The difference between the computed result and that of the reference is $5 \%$. The second solution is for a homogeneous transversely isotropic case, with the material symmetry axis specified coincident with the direction $y$ for both subdomains (results labeled $90^{\circ} / 0^{\circ} / 90^{\circ}-90^{\circ} / 0^{\circ} / 90^{\circ}$ in Figure 12). Once again, and as was expected, the computed $J$ values are constant along the complete crack front. In the third case the orientation of the material axes of symmetry are different in each subdomain: for subdomain I the material axis of symmetry is oriented in the $z$-direction, while for subdomain II it is oriented in the $y$-direction (results labeled $90^{\circ} / 90 / 0^{\circ}-90^{\circ} / 0^{\circ} / 90^{\circ}$ in Figure 12). The 


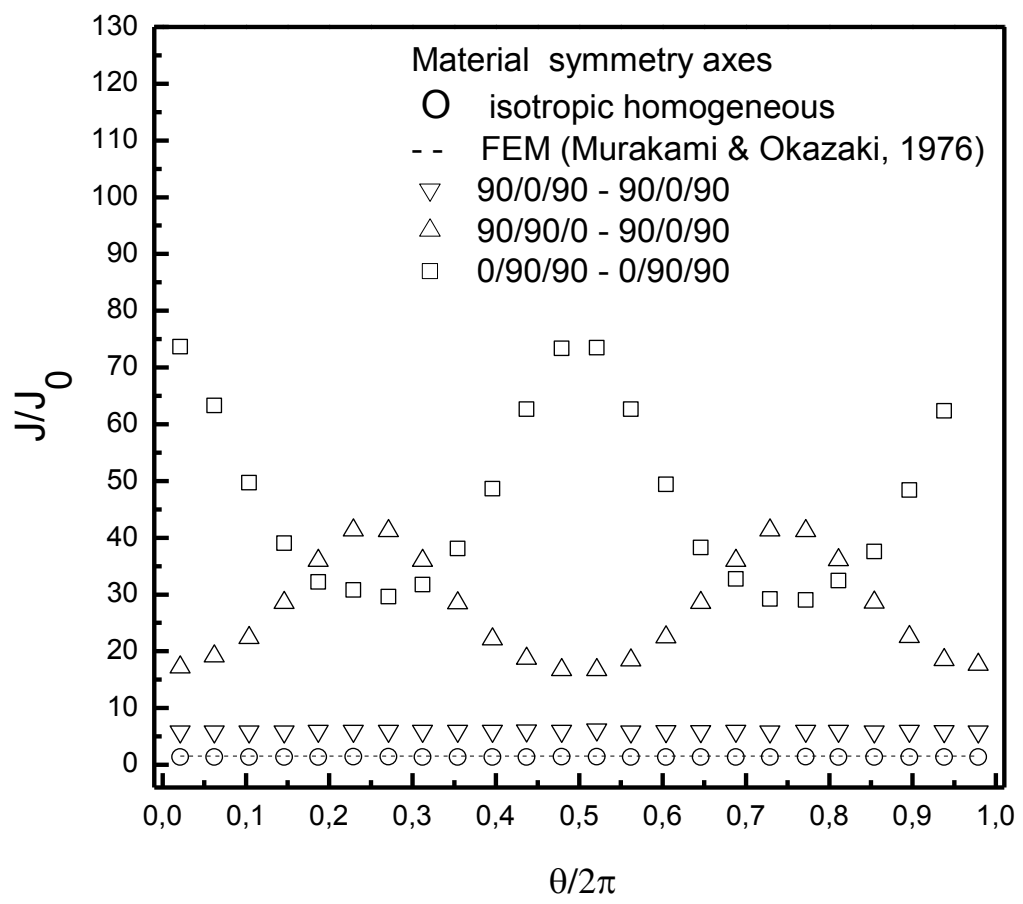

Figure 12. Normalized $J$-integral results along the crack front of the circumferential crack in the biomaterial bar.

$J$ results exhibit a periodic variation along the crack front. The minimum values occur in the positions coincident with the direction of the $z$-axis, while the maximum values are in the positions coincident with the direction of the $x$-axis. In the last case, the orientation of the material axis of symmetry is specified in the $x$-direction for both subdomains (results labeled $0^{\circ} / 90 / 90^{\circ}-0^{\circ} / 90^{\circ} / 90^{\circ}$ in Figure 12 ). As for the previous case, the $J$ results exhibit a periodic variation along the crack front. However, in this case minimum values occur in the positions coincident with the direction of the $x$-axis, while the maximums are in the positions coincident with the direction of the $z$-axis.

\section{Conclusions}

A boundary element implementation of the energy domain integral (EDI) for the computation of the $J$-integral of three-dimensional interface cracks in transversely isotropic bimaterials has been presented in this paper. The analysis is addressed using a multidomain boundary element method (BEM) formulation in order to account for the different material properties at both sides of the crack. The $J$-integral computation is implemented as a postprocessing technique, and so it can be applied to the results from a particular model at a later stage. The BEM uses a versatile set of fundamental solutions given only in terms of real functions which are valid for all possible material configurations. The implementation takes advantage of the efficiency of the boundary integral equation to directly obtain the required displacement derivatives, stress, and strain fields from their boundary integral representations.

The efficiency and accuracy of the proposed implementation has been addressed by analyzing a number of examples with straight and curved crack fronts. The computed results compare very well with those 
reported in the literature for benchmark problems. In addition, the implemented algorithm allowed for studying the effect of the relative orientations of the materials on both sides of the crack on the $J$ integral values.

Maximum errors and dependence of the computed results on the integration paths occur for surface cracks at the intersection of the crack front with a free surface. This behavior is attributed to the boundary layer effect taking place at the intersection of the crack front and a free surface. Under these circumstances, the EDI is not strictly applicable. This problem remains unsolved in this work. Following previous work [Ortiz and Cisilino 2005], alternative approaches for the selection of the auxiliary function $q$ for the implementation of the EDI could be explored to improve the accuracy of the computations.

\section{Appendix}

Additional terms of the components of the tensor $H(\hat{x})$ :

$$
\begin{aligned}
\beta_{3}=\left\{\frac{C_{44} c^{2}+C_{33} s^{2}}{C_{66}}\right\}^{1 / 2}, \quad h=\left\{c^{4}+\frac{\eta s^{2} c^{2}}{C_{11} C_{44}}+\frac{C_{33} s^{4}}{C_{11}}\right\}^{1 / 2}, \quad g=\left\{2\left(h+c^{2}\right)+\frac{\eta s^{2}}{C_{11} C_{44}}\right\}^{1 / 2}, \\
\xi=g\left(h+g \beta_{3}+\beta_{3}^{2}\right), \quad \eta=C_{11} C_{33}-C_{13}^{2}-2 C_{13} C_{44}, \quad f=\frac{h+c^{2}}{C_{66}}+\frac{g h}{C_{66} \beta_{3}}+\frac{C_{33} s^{2}}{C_{11} C_{44}},
\end{aligned}
$$

where $c=\cos \phi=x_{3} / r, s=\sin \phi=r_{12} / r$, the angle $\phi$ being as in Figure 4 .

\section{References}

[ABAQUS 2009] ABAQUS, Version 6.9-1, Dassault Systèmes, Providence, RI, 2009.

[Aliabadi 2002] M. H. Aliabadi, The boundary element method, 2: Applications in solids and structures, Wiley, Chichester, 2002.

[Anderson 2005] T. L. Anderson, Fracture mechanics: fundamentals and applications, 3rd ed., CRC Press, Boca Raton, FL, 2005.

[Ariza and Dominguez 2004a] M. P. Ariza and J. Dominguez, "Dynamic BE analysis of 3-D cracks in transversely isotropic solids", Comput. Methods Appl. Mech. Eng. 193:9-11 (2004), 765-779.

[Ariza and Dominguez 2004b] M. P. Ariza and J. Dominguez, "Boundary element formulation for 3D transversely isotropic cracked bodies", Int. J. Numer. Methods Eng. 60:4 (2004), 719-753.

[Balderrama et al. 2006] R. Balderrama, A. P. Cisilino, and M. Martinez, "Boundary element method analysis of threedimensional thermoelastic fracture problems using the energy domain integral", J. Appl. Mech. (ASME) 73:6 (2006), 959-969.

[Balderrama et al. 2008] R. Balderrama, A. P. Cisilino, and M. Martinez, "Boundary element analysis of three-dimensional mixed-mode thermoelastic crack problems using the interaction and the energy domain integrals", Int. J. Numer. Methods Eng. 74:2 (2008), 294-320.

[Boniface and Banks-Sills 2002] V. Boniface and L. Banks-Sills, "Stress intensity factors for finite interface cracks between a special pair of transversely isotropic materials", J. Appl. Mech. (ASME) 69:3 (2002), 230-239.

[Chen et al. 2009] C.-S. Chen, C.-H. Chen, and E. Pan, "Three-dimensional stress intensity factors of a central square crack in a transversely isotropic cuboid with arbitrary material orientations", Eng. Anal. Bound. Elem. 33:2 (2009), 128-136.

[Chow and Atluri 1998] W. T. Chow and S. N. Atluri, "Stress intensity factors as the fracture parameters for delamination crack growth in composite laminates", Comput. Mech. 21:1 (1998), 1-10.

[Chu and Hong 1990] S. J. Chu and C. S. Hong, "Application of the $J_{k}$ integral to mixed mode crack problems for anisotropic composite laminates", Eng. Fract. Mech. 35:6 (1990), 1093-1103.

[Cisilino and Aliabadi 1999] A. P. Cisilino and M. H. Aliabadi, "BEM implementation of the energy domain integral for the elastoplastic analysis of 3D fracture problems”, Int. J. Fract. 96:3 (1999), 229-245. 
[Cisilino et al. 1998] A. P. Cisilino, M. H. Aliabadi, and J. L. Otegui, "Energy domain integral applied to solve center and double-edge crack problems in three dimensions", Theor. Appl. Fract. Mech. 29:3 (1998), 181-194.

[Comninou 1977] M. Comninou, “The interface crack”, J. Appl. Mech. (ASME) 44:4 (1977), 631-636.

[Feijoó et al. 2000] R. A. Feijoó, C. Padra, R. Saliba, E. Taroco, and M. J. Vénere, "Shape sensitivity analysis for energy release rate evaluation and its application to the study of three-dimensional cracked bodies", Comput. Methods Appl. Mech. Eng. 188:4 (2000), 649-664.

[Freed and Banks-Sills 2005] Y. Freed and L. Banks-Sills, "A through interface crack between a $\pm 45^{\circ}$ transversely isotropic pair of materials", Int. J. Fract. 133:1 (2005), 1-41.

[Gibson 2007] R. F. Gibson, Principles of composite material mechanics, 2nd ed., CRC Press, Boca Raton, FL, 2007.

[He et al. 1994] W. J. He, J. E. Bolander, Jr., D. S. Lin, and H. J. Ding, "A boundary element for crack analysis at a bimaterial interface", Eng. Fract. Mech. 49:3 (1994), 405-410.

[Lachat and Watson 1976] J. C. Lachat and J. O. Watson, "Effective numerical treatment of boundary integral equations: a formulation for three-dimensional elastostatics", Int. J. Numer. Methods Eng. 10:5 (1976), 991-1005.

[Lifshitz and Rozentsveig 1947] I. M. Lifshitz and L. N. Rozentsveig, "Construction of the Green tensor for the fundamental equation of elasticity theory in the case of an unbounded elastically anisotropic medium”, Zh. Èksper. Teor. Fiz. 17 (1947), 783-791.

[Loloi 2000] M. Loloi, "Boundary integral equation solution of three-dimensional elastostatic problems in transversely isotropic solids using closed-form displacement fundamental solutions", Int. J. Numer. Methods Eng. 48:6 (2000), 823-842.

[Mao and Sun 1995] R. Mao and G. Sun, "A study of the interaction between matrix crack and matrix-fibre interface", Eng. Fract. Mech. 51:3 (1995), 469-477.

[Moran and Shih 1987] B. Moran and C. F. Shih, "A general treatment of crack tip contour integrals", Int. J. Fract. 35:4 (1987), 295-310.

[Murakami and Okazaki 1976] Y. Murakami and Y. Okazaki, "A simple procedure for the accurate determination of stress intensity factors by finite element method”, Trans. Jpn. Soc. Mech. Eng. 42:364 (1976), 3679-3687.

[Nahta and Moran 1993] R. Nahta and B. Moran, "Domain integrals for axisymmetric interface crack problems", Int. J. Solids Struct. 30:15 (1993), 2027-2040.

[Nikishkov and Atluri 1987] G. P. Nikishkov and S. N. Atluri, "Calculation of fracture mechanics parameters for an arbitrary three-dimensional crack, by the 'equivalent domain integral' method", Int. J. Numer. Methods Eng. 24:9 (1987), 1801-1821.

[Ortiz and Cisilino 2005] J. E. Ortiz and A. P. Cisilino, "Boundary element method for $J$-integral and stress intensity factor computations in three-dimensional interface cracks", Int. J. Fract. 133:3 (2005), 197-222.

[Pan and Chou 1976] Y.-C. Pan and T.-W. Chou, "Point force solution for an infinite transversely isotropic solid", J. Appl. Mech. (ASME) 43:4 (1976), 608-612.

[Rice 1968] J. R. Rice, "A path independent integral and the approximate analysis of strain concentration by notches and cracks", J. Appl. Mech. (ASME) 35:2 (1968), 379-386.

[Rice 1988] J. R. Rice, "Elastic fracture mechanics concepts for interfacial cracks.", J. Appl. Mech. (ASME) 55:1 (1988), 98-103.

[Sáez et al. 1997] A. Sáez, M. P. Ariza, and J. Dominguez, "Three-dimensional fracture analysis in transversely isotropic solids", Eng. Anal. Bound. Elem. 20:4 (1997), 287-298.

[Shah et al. 2006] P. D. Shah, C. L. Tan, and X. Wang, "Evaluation of T-stress for an interface crack between dissimilar anisotropic materials using the boundary element method", Comput. Model. Eng. Sci. 13:3 (2006), 185-198.

[So et al. 2004] W. M. G. So, K. J. Lau, and S. W. Ng, "Determination of stress intensity factors for interfacial cracks using the virtual crack extension approach", Comput. Model. Eng. Sci. 5:3 (2004), 189-200.

[Tan and Gao 1990] C. L. Tan and Y. L. Gao, "Treatment of bimaterial interface crack problems using the boundary element method", Eng. Fract. Mech. 36:6 (1990), 919-932.

[Távara et al. 2008] L. Távara, J. E. Ortiz, V. Mantič, and F. París, "Unique real-variable expressions of displacement and traction fundamental solutions covering all transversely isotropic elastic materials for 3D BEM", Int. J. Numer. Methods Eng. 74:5 (2008), 776-798. 
[Ting 1996] T. C.-T. Ting, Anisotropic elasticity: theory and applications, Oxford Engineering Science Series 45, Oxford University Press, New York, 1996.

[Zhao et al. 2007] M. H. Zhao, C. Y. Fan, T. Liu, and F. Yang, "Extended displacement discontinuity Green's functions for threedimensional transversely isotropic magneto-electro-elastic media and applications”, Eng. Anal. Bound. Elem. 31:6 (2007), $547-558$.

Received 19 Jun 2010. Revised 11 Dec 2010. Accepted 4 Feb 2011.

Nicolás O. LARRosa: nlarrosa@us.es

Departamento de Ingeniería Mecánica y de los Materiales, Escuela Técnica Superior de Ingeniería, Universidad de Sevilla, Camino Descubrimientos, s/n, 41092 Sevilla, Spain

JHONNY E. ORTIZ: jortiz@us.es

Departamento de Mecánica de Medios Continuos, Escuela Técnica Superior de Ingeniería, Universidad de Sevilla, Camino Descubrimientos, s/n, 41092 Sevilla, Spain

ADRIÁN P. CISILINO: cisilino@fi.mdp.edu.ar

División Soldadura y Fractomecánica, Instituto de Investigaciones en Ciencia y Tecnología de Materiales, Facultad de Ingeniería, Universidad Nacional de Mar del Plata - Consejo Nacional de Investigaciones Científicas, Av. Juan B. Justo 4302, B7608FDQ Mar del Plata, Argentina 


\title{
JOURNAL OF MECHANICS OF MATERIALS AND STRUCTURES
}

\author{
jomms.org
}

Founded by Charles R. Steele and Marie-Louise Steele

EDITORS

Charles R. SteEle

DAVIDE BIGONI

Stanford University, USA

YASUHIDE SHINDO

University of Illinois at Urbana-Champaign, USA

Tohoku University, Japan

\section{EDITORIAL BOARD}

$\begin{aligned} \text { H. D. BUI } & \text { École Polytechnique, France } \\ \text { J. P. CARTER } & \text { University of Sydney, Australia } \\ \text { R. M. CHRISTENSEN } & \text { Stanford University, USA } \\ \text { G. M. L. GLADWELL } & \text { University of Waterloo, Canada } \\ \text { D. H. HODGES } & \text { Georgia Institute of Technology, USA } \\ \text { J. HUTCHINSON } & \text { Harvard University, USA } \\ \text { C. HWU } & \text { National Cheng Kung University, Taiwan } \\ \text { B. L. KARIHALOO } & \text { University of Wales, UK } \\ \text { Y. Y. KIM } & \text { Seoul National University, Republic of Korea } \\ \text { Z. MROZ } & \text { Academy of Science, Poland } \\ \text { D. PAMPLONA } & \text { Universidade Católica do Rio de Janeiro, Brazil } \\ \text { M. B. RUBIN } & \text { Technion, Haifa, Israel } \\ \text { A. N. SHUPIKOV } & \text { Ukrainian Academy of Sciences, Ukraine } \\ \text { T. TARNAI } & \text { University Budapest, Hungary } \\ \text { F. Y. M. WAN } & \text { University of California, Irvine, USA } \\ \text { P. WRIGGERS } & \text { Universität Hannover, Germany } \\ \text { W. YANG } & \text { Tsinghua University, China } \\ \text { F. ZIEGLER } & \text { Technische Universität Wien, Austria } \\ & \\ \text { PRODUCTION } & \text { contact@ msp.org } \\ \text { SILVIO LEVY } & \text { Scientific Editor }\end{aligned}$

Cover design: Alex Scorpan

Cover photo: Mando Gomez, www.mandolux.com

See http://jomms.org for submission guidelines.

JoMMS (ISSN 1559-3959) is published in 10 issues a year. The subscription price for 2011 is US \$520/year for the electronic version, and \$690/year (+\$60 shipping outside the US) for print and electronic. Subscriptions, requests for back issues, and changes of address should be sent to Mathematical Sciences Publishers, Department of Mathematics, University of California, Berkeley, CA 94720-3840.

JoMMS peer-review and production is managed by EditFLow ${ }^{\circledR}$ from Mathematical Sciences Publishers.

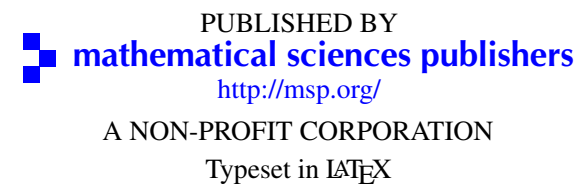

Copyright (C2011 by Mathematical Sciences Publishers 


\section{Journal of Mechanics of Materials and Structures}

\section{Volume 6, No. 7-8}

September-October 2011

\section{Special issue \\ Eleventh Pan-American Congress \\ of Applied Mechanics (PACAM XI)}

Preface

Adair R. Aguiar

949

Influence of specimen geometry on the Portevin-Le Châtelier effect due to dynamic strain aging

for the AA5083-H116 aluminum alloy

Rodrigo Nogueira de Codes and Ahmed Benallal

Dispersion relations for SH waves on a magnetoelectroelastic heterostructure with imperfect

interfaces

J. A. Otero, H. Calas, R. Rodríguez, J. Bravo, A. R. Aguiar and G. Monsivais

Numerical linear stability analysis of a thermocapillary-driven liquid bridge with magnetic stabilization

Yue Huang and Brent C. Houchens

Numerical investigation of director orientation and flow of nematic liquid crystals in a planar 1:4 expansion Pedro a. Cruz, Murilo F. Tomé, IAin W. Stewart and Sean McKee

Critical threshold and underlying dynamical phenomena in pedestrian-induced lateral vibrations of footbridges

Stefano LenCI and LAURA MARCHEgGiani

Free vibration of a simulation CANDU nuclear fuel bundle structure inside a tube

XUAN ZHANG and SHUdONG Yu

Nonlinear dynamics and sensitivity to imperfections in Augusti's model

D. Orlando, P. B. Gonçalves, G. Rega and S. LenCi

Active control of vortex-induced vibrations in offshore catenary risers: A nonlinear normal mode approach

CArlos E. N. MAZzilli and César T. SANCheS

Nonlinear electromechanical fields and localized polarization switching of piezoelectric macrofiber composites

Yasuhide Shindo, Fumio Narita, KoJi SATo and Tomo TAKeda

1089

Three-dimensional BEM analysis to assess delamination cracks between two transversely isotropic materials

Nicolás O. Larrosa, Jhonny E. Ortiz and Adrián P. Cisillino

Porcine dermis in uniaxial cyclic loading: Sample preparation, experimental results and modeling

A. E. Ehret, M. Hollenstein, E. MAzzA and M. Itskov

Analysis of nonstationary random processes using smooth decomposition

Rubens SAMpaio and Sergio Bellizzi

Perturbation stochastic finite element-based homogenization of polycrystalline materials

S. LePage, F. V. Stump, I. H. Kim and P. H. Geubelle

A collocation approach for spatial discretization of stochastic peridynamic modeling of fracture

Georgios I. Evangelatos and POL D. SPANOS 